\title{
Cytomegaloviral Retinitis
}

National Cancer Institute

\section{Source}

National Cancer Institute. Cytomegaloviral Retinitis. NCI Thesaurus. Code C50521.

Inflammation of the retina due to cytomegalovirus. 